Provided for non-commercial research and education use. Not for reproduction, distribution or commercial use.

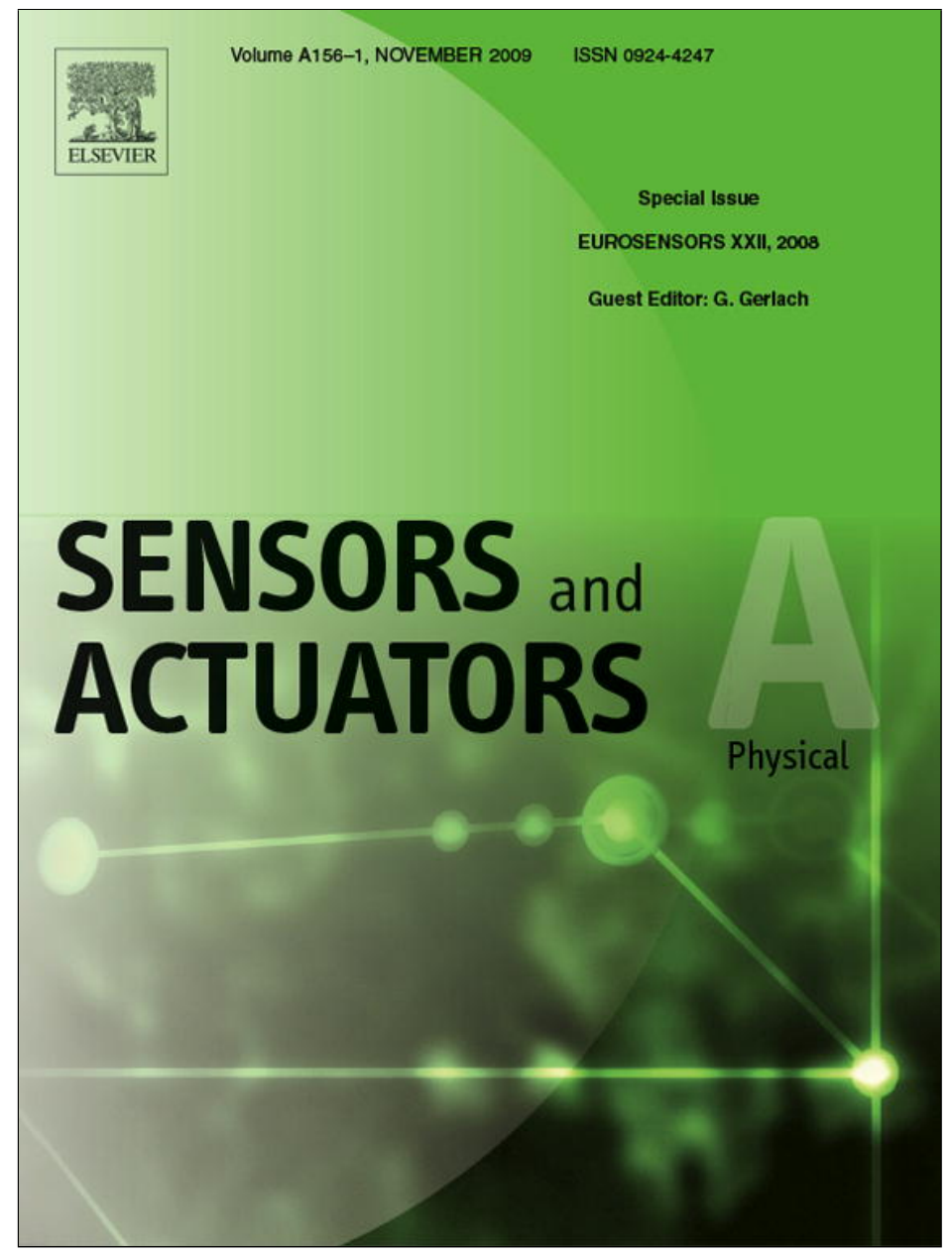

This article appeared in a journal published by Elsevier. The attached copy is furnished to the author for internal non-commercial research and education use, including for instruction at the authors institution and sharing with colleagues.

Other uses, including reproduction and distribution, or selling or licensing copies, or posting to personal, institutional or third party websites are prohibited.

In most cases authors are permitted to post their version of the article (e.g. in Word or Tex form) to their personal website or institutional repository. Authors requiring further information regarding Elsevier's archiving and manuscript policies are encouraged to visit:

http://www.elsevier.com/copyright 


\title{
An integrated vision system with autofocus for wireless capsular endoscopy
}

\author{
C. Cavallotti ${ }^{\mathrm{a}, *}$, M. Piccigallo ${ }^{\mathrm{a}}$, E. Susilo ${ }^{\mathrm{a}, \mathrm{b}}$, P. Valdastri $^{\mathrm{a}}$, A. Menciassi $^{\mathrm{a}, \mathrm{b}}$, Paolo Dario ${ }^{\mathrm{a}, \mathrm{b}}$ \\ a CRIM Lab, Polo Sant'Anna Valdera, Scuola Superiore Sant'Anna, 56025 Pontedera, Pisa, Italy \\ ${ }^{\mathrm{b}}$ Italian Institute of Technology Network, Genova, Italy
}

\section{A R T I C L E I N F O}

\section{Article history:}

Received 27 September 2008

Received in revised form

22 November 2008

Accepted 25 January 2009

Available online 9 March 2009

Keywords:

Autofocus

Liquid lens

Capsule endoscopy

\begin{abstract}
A B S T R A C T
A prototype of a vision system for capsular endoscopy provided with autofocus function was developed. It is composed by two main parts: a wireless capsule with an adjustable focus in the range between $15 \mathrm{~mm}$ and $100 \mathrm{~mm}$, and a personal computer where the autofocus algorithm is implemented. Several benches and ex vivo tests were performed in order to validate the developed system and encouraging results were obtained. Further miniaturization will be pursued to obtain a swallowable device adequate for capsular endoscopy procedures.
\end{abstract}

(C) 2009 Elsevier B.V. All rights reserved.

\section{Introduction}

A significant advancement in the field of medical devices was represented by the introduction of the wireless capsule endoscopy (WCE), a medical procedure that has enabled, for the first time, a painless diagnosis inside the gastrointestinal (GI) tract with bright perspectives for screening [1]. This technique has allowed to detect abnormalities of the small intestine and shows great promise in reducing invasiveness of gastroscopy and colonoscopy.

WCE takes advantage from swallowable devices capable of acquiring images of gastrointestinal mucosa through an optical lens and a CMOS or CCD image sensor, a LED-based illumination system ( 2 or more LEDs), a battery as energy source and an electronic device capable of transmitting the data to a recorder through some receivers attached on the patient's abdomen. Depending on the specific platform, images can be viewed in real time or stored for later diagnosis [2,3]. A list of relevant WCE platforms is reported in Table 1, where all the main features for each system are highlighted. One of the main limitations common to all those WCE systems is the passive locomotion. In particular, the endoscopic pills, relying just on peristalsis, are forced to go forward without the possibility to stop, turn or go back during their journey inside the GI tract. Furthermore, all the available WCE platforms have a fixed focal length. This can be acceptable considering that the lumen is collapsed, thus the distance between the camera in the pill and the target, i.e. the collapsed lumen wall, is almost constant during the whole capsule journey along the GI tract.

\footnotetext{
* Corresponding author.

E-mail address: c.cavallotti@sssup.it (C. Cavallotti).
}

Several research groups worldwide are trying to develop active locomotion systems to be embedded into WCE. This would allow to perform endoscopy in a totally controlled manner, being the doctor able to steer the capsule towards the more interesting pathological areas [4]. Examples of active locomotion in WCE range from legged or crawling locomotion [5,6], to external magnetic steering [7]. In this scenario, images obtained by the vision system would be useful either for the purpose of diagnostics or for autonomous steering of the capsule, e.g. facing the lumen or holding a given position against the natural bowel movements. Therefore, a real time image stream has to be processed for identification of visual clues that, together with position information, can be used as high-level input to the locomotion controller. Sharp images are required in order to achieve this goal. Therefore an autofocus system would enable a reliable implementation of this feature.

Another application of WCE where an autofocus system would dramatically improve the procedure outcomes relates to a novel approach for gastric screening. As reported in [8], one liter of transparent fluid, either water or polyethilenglycole (PEG), may be ingested by the patient undergoing the procedure. This liquid stays in the stomach for roughly half hour, thus allowing tissue distension and enabling underwater capsular endoscopy. Given the size of the gastric cavity, the capsule may stay between $3 \mathrm{~mm}$ to 100 $\mathrm{mm}$ away from the stomach mucosa. In this scenario, having an on-board autofocus system would enable the acquisition of sharp images wherever the capsule is located. Furthermore, considering an active propulsion system for WCE in a liquid environment, as proposed in [9], the autofocus would enable autonomous steering and locomotion.

Conventional optical systems with adjustable focus consist of a group of optical lenses and at least one actuator to vary lens 
Table 1

Characteristic of various endoscopic capsule

\begin{tabular}{|c|c|c|c|c|c|c|c|}
\hline & Operative environment & Optical sensor & Dimensions $(L \times D)$ & Frame rate & $\begin{array}{l}\text { Source of } \\
\text { motion }\end{array}$ & Focal length & $\begin{array}{l}\text { Real time } \\
\text { viewer }\end{array}$ \\
\hline $\begin{array}{l}\text { GIVEN IMAGING (Pillcam SB, } \\
\text { ESO, COLON) }\end{array}$ & $\begin{array}{l}\text { Small intestine, } \\
\text { esophagus, colon }\end{array}$ & CMOS & $11 \mathrm{~mm} \times 26 \mathrm{~mm}$ & $2-7-14$ image/s & Peristalsis & Fixed & No \\
\hline OLYMPUS (EndoCapsule) & Small intestine & CCD & $11 \mathrm{~mm} \times 26 \mathrm{~mm}$ & 2 image/s & Peristalsis & Fixed & Yes \\
\hline JINSHAN GROUP (Omom) & Small intestine & CMOS & $13 \mathrm{~mm} \times 27.9 \mathrm{~mm}$ & 2 image/s & Peristalsis & Fixed & No \\
\hline INTROMEDIC (Miro) & Small intestine & CMOS & $10.8 \mathrm{~mm} \times 24 \mathrm{~mm}$ & $1.4-2.8$ image $/ \mathrm{s}$ & Peristalsis & Fixed & No \\
\hline
\end{tabular}

position and magnification. An interesting example of a miniature camera robot for abdominal exploration implementing mechanical focus adjustment is presented in [10]. An electromagnetic brushless motor is directly connected to a lead screw, which is linked to a slider, where a lens is placed. By actuating the motor, the distance between the lens and the image sensor can be varied.

Similar systems, together with image processing through a proper sharpness function, can be used to implement autofocus. By spanning the actuator through all its working range, it is possible to evaluate a sharpness function for each lens position. The maximum output value corresponds to the focused image, thus the actuator is driven back to the corresponding position, in order to finally obtain a sharp image. Precision and hysteresis in lens positioning severely affect the performance of an autofocus system, therefore extreme care must be devoted in avoiding those effects. Another important requirement is the fast response of the actuator, especially for autonomous locomotion. Stepper, ultrasonic and voice-coil motors are used as actuators in traditional autofocus systems $[11,12]$. The main drawback for the stepper motor is related to the quite large volume required, that would not fit the dimensional constraints of endoscopic capsules. Voice-coil motors have fast response and low cost, but present a relevant hysteresis due mainly to the mechanical moving parts. In the recent past, many efforts have been addressed to obtain variable focusing microlenses or microlens systems without any mechanical moving part to be integrated inside mobile phones or slim digital cameras. This would enable a reduction in terms of space and price, while increasing autofocus speed $[13,14]$. Existing approaches include pneumatic [15], piezoelectric [16] or thermal actuation [17], liquid cristal [18] and electrowetting $[13,14,19-21]$. Recently, it was shown that liquid lenses can be used to set up reliable and fast focusing systems for digital cameras [13] due to simple implementation, small electrical dissipation and limited size. A liquid lens is a cell which contains two immiscible liquids, one based on a water solution, while the other is apolar and non-conductive. Liquids are transparent and have the same density. The natural interface between the two liquids forms a natural diopter, due to the refractive index difference. The working principle of a liquid lens is based on the electrowetting phenomenon, i.e. application of a voltage between the electrode deposited on the external side of the cell and the conducting liquid deforms the interface between the two liquids, thus changing the focal length $[13,19]$.

In the present study the authors developed a wireless capsule with an adaptable optical focus based on a liquid lens. A system overview and the optical design are given in Section 2.1. An autofocus algorithm based on a purposely selected sharpening function is described in Section 2.2. Experimental results and conclusions are reported in Sections 3 and 4, respectively.

\section{Materials and methods}

\subsection{System overview}

As represented in Fig. 1, the proposed system is composed by two main parts, a wireless capsule with an adjustable focus system and a personal computer (PC), where the autofocus algorithm is implemented. The wireless capsule, schematically represented in Fig. 2, embeds a vision module with a liquid lens, a dedicated high data rate (HDR) transmitter, an illumination submodule, a wireless microcontroller and a small battery pack.

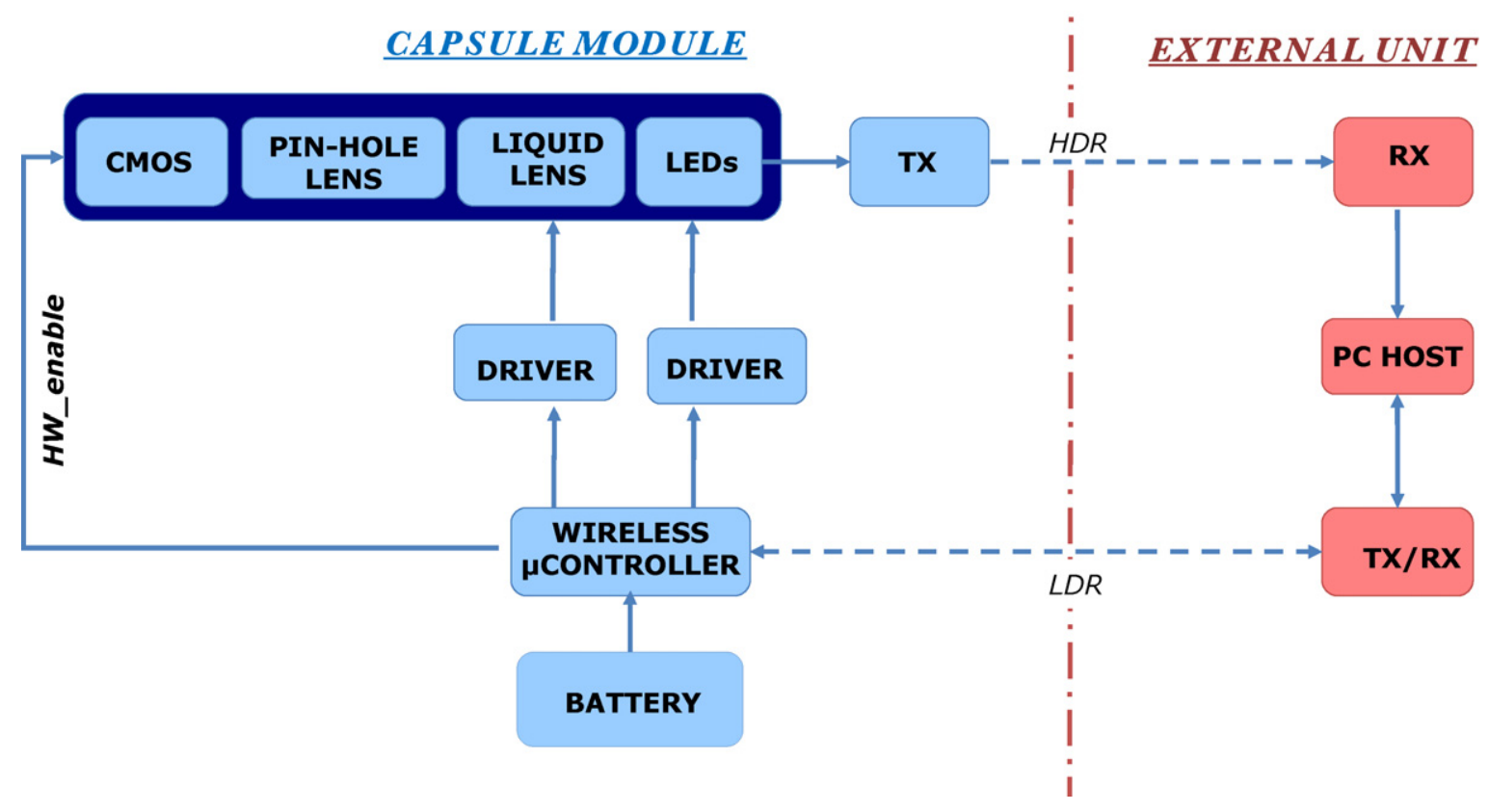

Fig. 1. Block diagram of the first wireless capsule prototype with autofocus feature. 


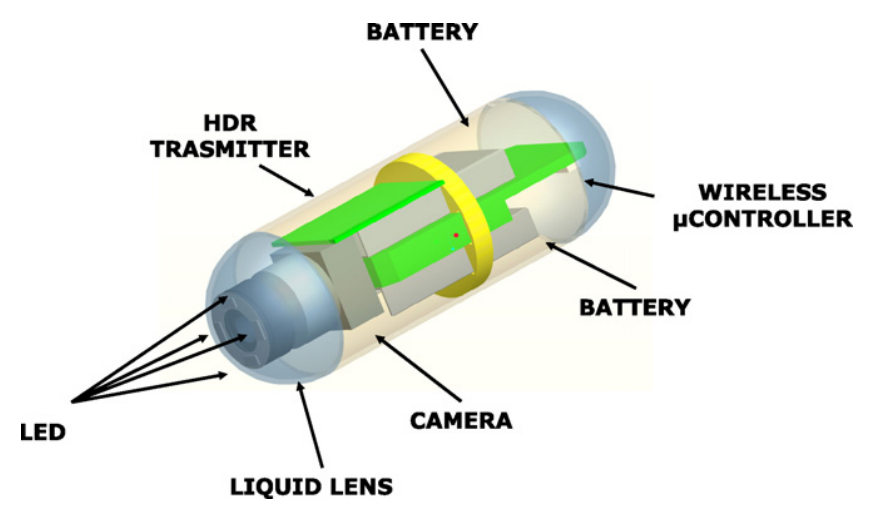

Fig. 2. Capsule design.

A commercial camera (MO-S588, Misumi Electronics Corp., Taiwan) was selected as the best trade-off between image quality and small package dimension $(8 \mathrm{~mm} \times 8 \mathrm{~mm} \times 9.5 \mathrm{~mm})$, available off-the-shelf. It embeds a VGA $(510 \times 492$ pixels $)$ CMOS sensor (OV7930, Omnivision, CA) with a pin-hole lens whose distance from the sensor is adjustable in a range from $3.1 \mathrm{~mm}$ to $3.5 \mathrm{~mm}$. A liquid lens (ARCTIC 416, Varioptic, Switzerland), placed in front of the pinhole, as shown in Fig. 3, allows the adjustment of the overall focal length. This lens has an outer diameter of $7.75 \mathrm{~mm}$ and a thickness of $2 \mathrm{~mm}$. The quantity $(1 / f)$, where $f$ is the focal length $(\mathrm{m})$, can be varied between -10 and 20 diopters $\left(\mathrm{m}^{-1}\right)$, thus obtaining a varying focal length in the range from $-\infty$ to $-100 \mathrm{~mm}$ and from $50 \mathrm{~mm}$ to $+\infty$. The value of $(1 / f)$ is linear with the root mean square (RMS) of the applied voltage $V$. A dedicated driving circuit is required to generate the control signal for the liquid lens. The one we selected (DrivBoard 60 LL3 PWM, Durel, USA) is based on a step-up converter and transforms a pulse width modulation (PWM) signal with a frequency of about $25 \mathrm{kHz}$ in a analogue control voltage. By varying the PWM duty cycle from $0 \%$ to $100 \%$, a RMS voltage ranging from $9 \mathrm{~V}$ to $65 \mathrm{~V}$ can be applied to the liquid lens. In order to have just a positive lens and a linear optic behavior, a voltage ranging from $36 \mathrm{~V}$ to $60 \mathrm{~V}$ must be imposed, corresponding to a duty cycle from $60 \%$ to $92 \%$. The overall optical system, shown in Fig. 3 is designed to achieve an autofocus range of about $90 \mathrm{~mm}$ for a target placed from $10 \mathrm{~mm}$ to $100 \mathrm{~mm}$ away from the capsule, which are typical operative distances for endoscopy in a distended stomach. For the purpose of achieving this focal range the distance $a$ was fixed to $3.3 \mathrm{~mm}$. Applying the thin lens formula, this value was obtained and it was further validated by bench testing.

The output data flow coming from the CMOS camera is directly linked to an HDR transmitter which enables real time streaming at a frame rate of $30 \mathrm{fps}$. The transmitter works with a carrier frequency from 900 to $1230 \mathrm{MHz}$. Its dimensions are: $10 \mathrm{~mm}(\mathrm{~h}) \times$ $0.4 \mathrm{~mm}(\mathrm{w}) \times 13.4 \mathrm{~mm}(\mathrm{l})$, Even if it can be further scaled down

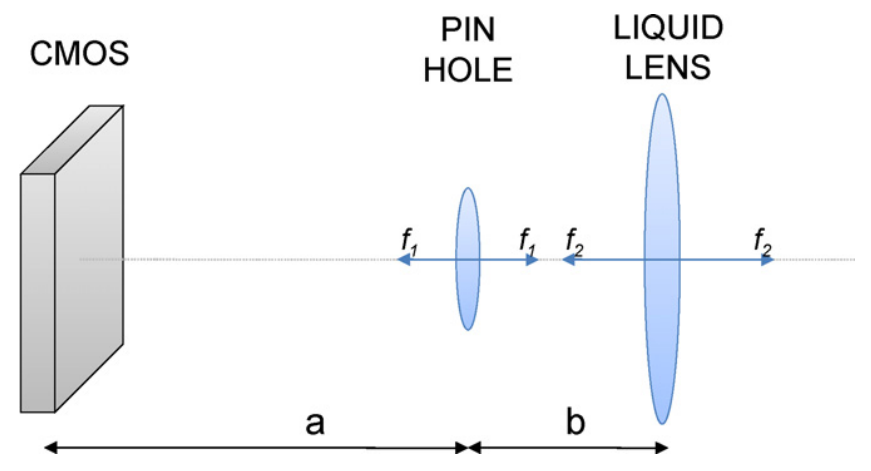

Fig. 3. Sketch of the optical design of the capsule system.

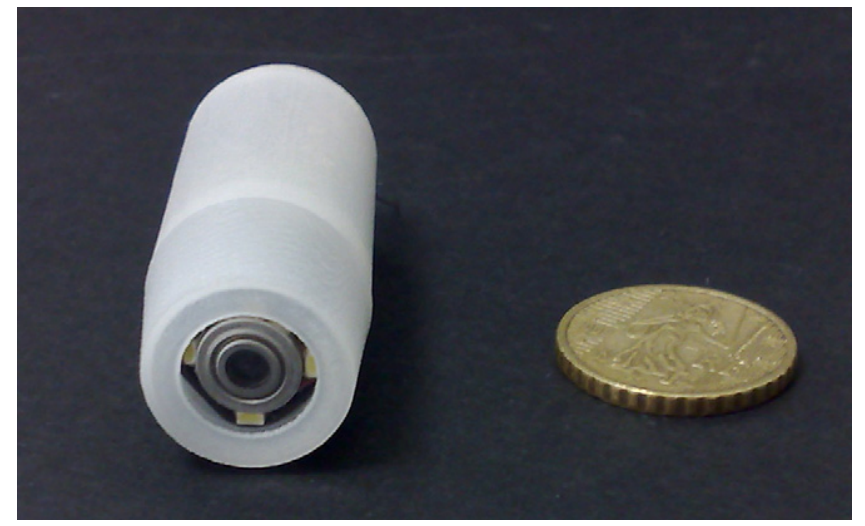

Fig. 4. Capsule prototype.

in dimension by adopting custom approaches [22]. The illumination submodule includes four white LED units (Nesw007AT, Nichia, Japan), arranged in a cross-shaped configuration around the camera and driven by a dedicated circuit (FAN5611, Fairchild, Maine). Both the current intensity for the illumination system and the focus length adjustment can be controlled by PWM digital signals generated by the on-board microcontroller (CC2430, Texas Instrument). This integrated circuit also embeds an IEEE 802.15.4 compliant transceiver, thus allowing a low data rate (LDR) bidirectional communication with an external unit. A commercially available board (RC2300, Radiocrafts, Norway), integrating the selected microcontroller, was used in order to reduce prototyping time. This solution demonstrated to be effective for in vivo WCE applications, as reported in [23], even if the board dimension can be further optimized, as presented in [24]. Regarding the batteries, we used rechargeable Li-Ion polymer cells (LP20, Plantraco, USA), having a nominal capacity of $20 \mathrm{mAh}$ and a size of $11 \mathrm{~mm}(\mathrm{~h}) \times 3 \mathrm{~mm}(\mathrm{w}) \times 12 \mathrm{~mm}(\mathrm{l})$. The overall dimension of the capsule, represented in Fig. 4, are $15 \mathrm{~mm}$ in diameter and $40 \mathrm{~mm}$ in length. As mentioned above, almost all the selected components can be reduced in dimensions by adopting customized solutions, thus achieving swallowable size, typically $11 \mathrm{~mm}(\mathrm{~d}) \times 30 \mathrm{~mm}(\mathrm{l})$.

The external part of the system is composed by a standard PC with a frame grabber, an HDR receiver and a LDR transceiver. This last unit has the same wireless microcontroller used in the capsule, which is connected to the PC via a serial to USB converter (FT232R, FTDI, UK). The HDR receiver is connected to a frame grabber (Cynergy XS, Terratec, Germany), that digitalizes the data stream coming from the camera pill. A purposely developed autofocus algorithm runs on the PC. It processes the image coming from the frame grabber, computing a sharpness function, and drives the liquid lens by setting the desired PWM parameters through the LDR wireless link. A user interface, implemented by Labview 8.2 (National Instruments, USA), allows the user to visualize the video stream, switch on and off the autofocus feature, adjust the illumination and monitor other relevant parameters coming from the capsule, such as the battery level.

\subsection{Autofocus algorithm}

An autofocus system is based on a sharpness function that is applied to images acquired by a camera at different values of focus. A peak on the value of the focus function means that the corresponding image is sharper than the others. Various sharpness functions have been introduced and studied in literature: in [25] and in [26] a comparison between them is reported. We applied some of the functions that achieved the best score to some videos acquired during in vivo tests performed on a $30 \mathrm{~kg}$ female pig in an authorized 


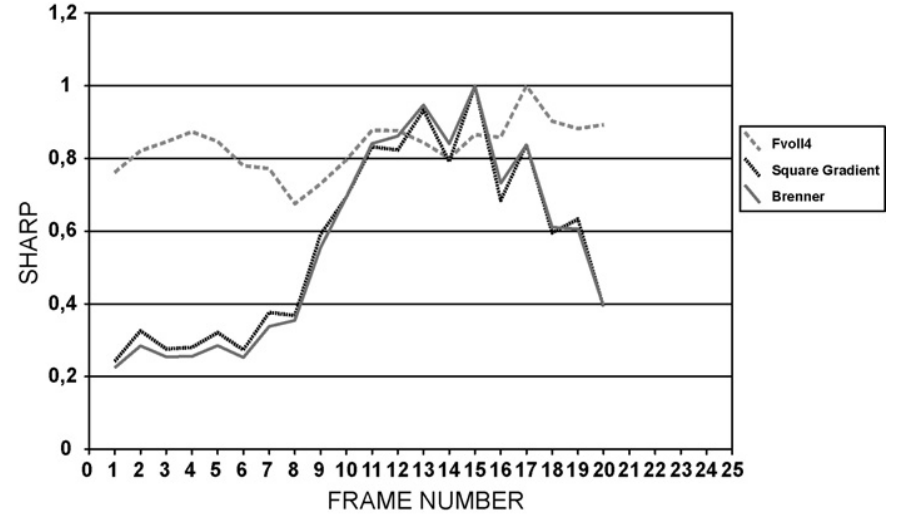

Fig. 5. Plot of the value of different focus functions for the same video frames.

laboratory, complying with the assistance and collaboration of a specially trained medical team, in accordance to all ethical considerations and the regulatory issues related to animal experiments. During these tests, the PWM signal driving the liquid lens varied from minimum to maximum, and a video was recorded in order to study a suitable focus function.

Some of the functions that were considered are the following:

(1) Square gradient function:

$$
F_{s q_{\text {_grad }}}=\sum_{i=1}^{M} \sum_{j=1}^{N}(g(i, j+1)-g(i, j))^{2}
$$

where $g(i, j)$ is the monochrome intensity of the pixel $(i, j)$. The function is based on the difference in intensity between two contiguous pixels, assuming that in a sharp image this difference is significant.

(2) Brenner function:

$$
F_{\text {brenner }}=\sum_{i=1}^{M} \sum_{j=1}^{N}(g(i, j+2)-g(i, j))^{2}
$$

where the difference is calculated between a pixel and its neighbor two points away [27].

(3) Vollath function:

$$
F_{\text {Voll } 4}=\sum_{i=1}^{M-1} \sum_{j=1}^{N} g(i, j) \cdot g(i+1, j)-\sum_{i=1}^{M-2} \sum_{j=1}^{N} g(i, j) \cdot g(i+2, j)
$$

it has very good performance in the presence of noise and it is based on the autocorrelation function [28].

A comparison among the above written functions applied to the frames of the video acquired during the in vivo test is shown in Fig. 5, where the values are normalized by the maximum value in order to compare the function shapes. As a criterion for judging a sharpness function, we chose the peak shape: if the peak of the function is well defined, then it is easier to find the focus position. It is clear that the choice of the function depends on the particular application. In our case the $F_{\text {brenner }}$ and the $F_{s q_{\text {grad }}}$ gave the best result, and they are not significantly different from each other. The results are in accordance with [26], but not with [25], where the Vollath function obtained the best score. The function chosen for the implementation of autofocus on the capsule is the $F_{s q_{-} \text {grad }}$, in accordance also to [29], because theoretically it gives a more marked difference between a perfectly sharp image and a defocused one. In order to obtain a smoother curve, and reduce the local maxima, a threshold was considered, so that the difference between two pixels is added to the sum only if it exceeds the threshold value. Other slight modifications have been introduced with the purpose of enhancing the peak. In particular, for each term of the sum (1), two adjacent pixels are considered not only along the $x$ axis, but also along the $y$ axis and the best of the two values is considered. In addition, all the three colors (blue, green and red) are considered to compute the pixel intensity value $g(i, j)$, then the best of the three values of difference between adjacent pixels is added to the total sum. The resulting sharpness function has a well-defined peak.

The function was calculated for a portion of the global image, i.e. a central window composed by $200 \times 200$ pixels ( $M$ value from 220 to 420 and $N$ from 120 to 320 ).

In order to find the maximum value of the function along the range, a peak-searching algorithm was implemented as follows.

As soon as the autofocus command is issued by the user on the interface, a loop starts: at each iteration a PWM on the capsule is set, an image is acquired and the corresponding sharpness function value (SHARP_ACTUAL) is evaluated. Then the PWM to be set at the next iteration is increased by a value STEPSIZE. SHARP_ACTUAL is compared to the value that the same function assumes for the image acquired at the first step (SHARP_FIRST). If SHARP_ACTUALSHARP_FIRST $>$ THRESHOLD (where THRESHOLD is a constant set at the beginning), the value of STEPSIZE is decreased in order to refine the search. When the value of SHARP_ACTUAL decreases, it means that a maximum (that could be a local maximum) is found, and in the subsequent iterations the value SHARP_ACTUAL

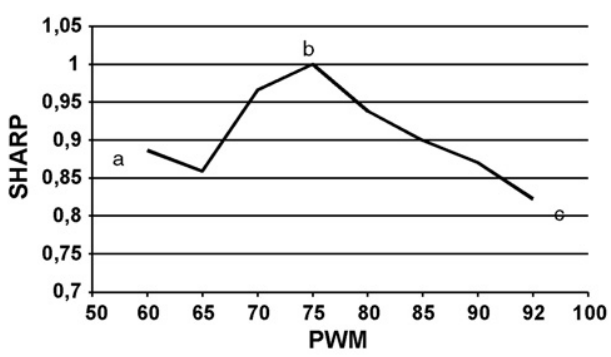

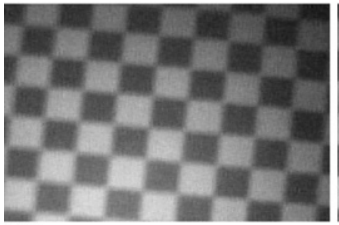

(a)

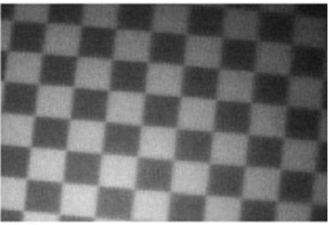

(b)

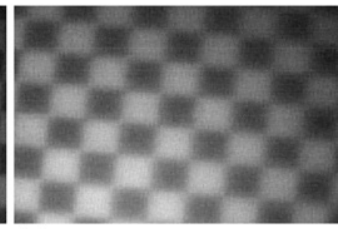

(c)

Fig. 6. Plot of the value of squared gradient function versus PWM values for the same scene. 


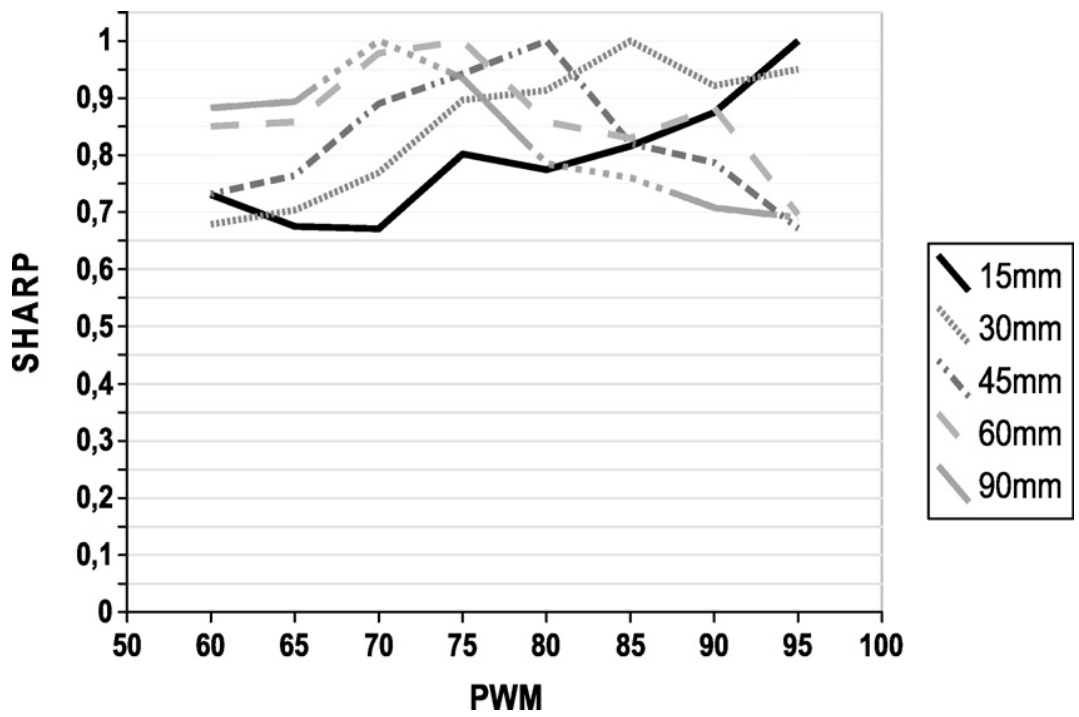

Fig. 7. Plot of the value of squared function for different object distances from camera.

is compared to the maximum value (SHARP_MAXIMUM) assumed by the sharpness function up to this point: if SHARP_MAXIMUMSHARP_ACTUAL >THRESHOLD, then the peak is found, thus the PWM is again set to the value corresponding to SHARP_MAXIMUM and STEPSIZE is decreased in order to refine the search around the maximum. When STEPSIZE reaches a predetermined minimum value, the loop exits, and PWM is set to the value that corresponds to the maximum value of the sharpness function, and thus to a focused image. According to the datasheets of the liquid lens and lens driver, the PWM can vary in a range between $60 \%$ and $92 \%$, that, mapped to a byte (256 values), corresponds to a range between 153 and 235. A step size of 3, 10 and 15 was considered.

\section{Experimental results}

In order to validate the proposed system, two different kinds of test were performed. A first set was aimed at investigating the autofocus system performances by testing the wireless capsule on standard images on bench. The second trial was performed on freshly harvested gastric porcine specimens, in order to evaluate the device in conditions that are as close as possible to the operative ones.
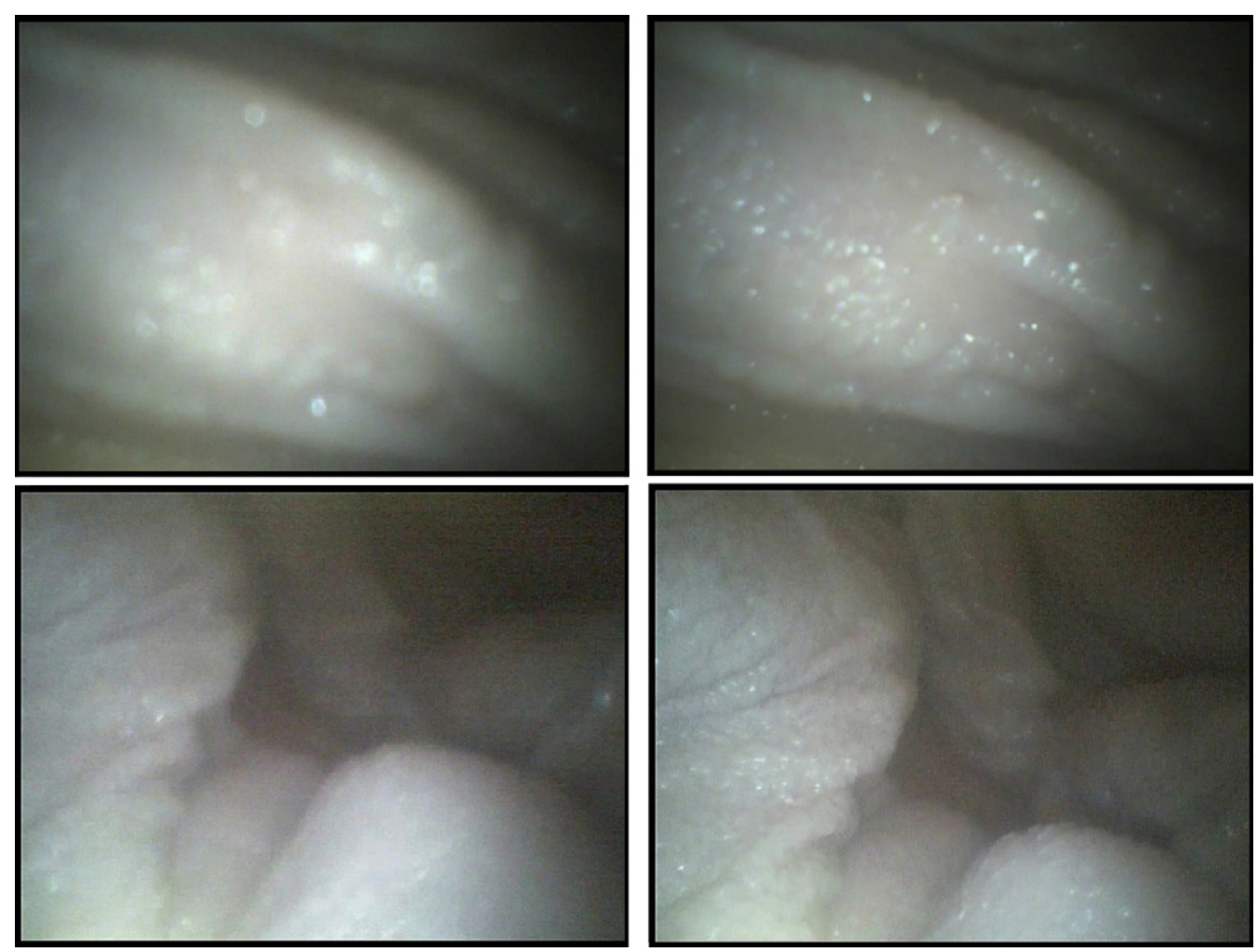

Fig. 8. Acquired images during ex vivo test before (left) and after (right) autofocus. 


\subsection{Bench tests}

For bench testing, a black and white grid, with a squared pattern of $3 \mathrm{~mm} \times 3 \mathrm{~mm}$, was used. It was positioned at a distance of $4 \mathrm{~cm}$ from the system and the autofocus algorithm was started. Fig. 6 shows the sharpness function versus PWM value and three images at three different PWM values. The second image represents the in-focus image: it was obtained with a duty cycle of $75 \%$. Then the grid was placed at different distances from the capsule in a range between $15 \mathrm{~mm}$ and $90 \mathrm{~mm}$. This translates in a shifting of the sharpness function peak on the PWM scale, clearly visible in Fig. 7 where the sharp values are normalized by the maximum value in order to compare the function shapes.

\subsection{Ex vivo tests}

A freshly harvested porcine stomach, having rough external dimensions of $15 \mathrm{~cm} \times 25 \mathrm{~cm}$, was distended by air insufflation, the two openings (pylorus and gastro esophageal junction) were sutured in order to minimize the air leakage and to have an insufflated stomach during the whole test. The wireless capsule, represented in Fig. 4 with dimension of $15 \mathrm{~mm}(\mathrm{~d}) \times 40 \mathrm{~mm}(\mathrm{l})$, was inserted in the stomach and was anchored on the tissue by magnetic force: a small permanent magnet, placed inside the capsule, is attracted by another magnet kept outside the stomach. Thus, the camera is fixed in a position and directed towards the opposite gastric wall. Several tests were performed with encouraging results. The autofocus algorithm works satisfactory; the acquired images are bright and clear, thanks to proper adjustment of focus and illumination (see Fig. 8).

\section{Discussion and conclusions}

A preliminary wireless capsule prototype with autofocus feature was developed with size of $15 \mathrm{~mm}$ (d) and $40 \mathrm{~mm}$ (1). It shows a field of view (FOV) of $86^{\circ}$ (horizontal) by $64^{\circ}$ (vertical) and a focus range between $15 \mathrm{~mm}$ and $100 \mathrm{~mm}$, respectively with the maximum and the minimum value of duty cycle. The capsule lifetime, for the first prototype, is about $10 \mathrm{~min}$, considering the battery and all the other components presented in Section 2.1. An autofocus algorithm was implemented on a PC in order to obtain a focused image. Focus time depends on the settling time of the lens and the delay in LDR transmission. The lens takes about $40 \mathrm{~ms}$ to reach its stable state and the delay in LDR transmission for each step is $30 \mathrm{~ms}$. Thus considering an average number of 20 steps to find the peak position, the focus time can be estimated equal to $1400 \mathrm{~ms}$.

As demonstrated in [30,14], liquid lenses do not show substantial hysteresis and do not affect the image quality of the camera. For the next prototype, a better power management will be investigated in order to increase the battery lifetime, and further miniaturization will be pursued in order to fabricate a swallowable device.

\section{Acknowledgment}

The work described in this paper was funded in part by the Intelligent Microsystem Center, KIST, South Korea, and in part by the European Commission in the framework of VECTOR FP6 European project EU/IST-2006-033970. The authors are also grateful to Prof. M.O. Schurr and all the team from novineon Healthcare Technology Partners GmbH, Tubingen, Germany, for the help during the testing phase of the device. They also thank F. Focacci for his valuable assistance.

\section{References}

[1] A. Moglia, A. Menciassi, M.O. Schurr, P. Dario, Wireless capsule endoscopy: from diagnostic devices to multipurpose robotic systems, Biomed. Microdevices 9 (2) (2007) 235-243.
[2] M. Waterman, R. Eliakim, Capsule enteroscopy of the small intestine, Abdom. Imaging, 25 June 2008.

[3] P. Swain, The future of wireless capsule endoscopy, World J Gastroenterol 14 (26) (2008) 4142-4145.

[4] M. Quirini, S. Scapellato, P. Valdastri, A. Menciassi, P. Dario, An approach to capsular endoscopy with active motion, in: Conf Proc IEEE Eng Med Biol Soc, 2007, pp. 2827-2830.

[5] M. Quirini, R. Webster, A. Menciassi, P. Dario, Design of a pill-sized 12-legged endoscopic capsule robotic, in: Robotics and Automation, 2007 IEEE International Conference, 2007, pp. 1856-1862.

[6] B. Kim, D.H. Kim, J. Jung, J.O. Park, A biomimetic undulatory tadpole robot using ionic polymer-metal composite actuators, Smart Mater. Struct. 14 (2005) 1585-1979.

[7] F. Carpi, S. Galbiati, A. Carpi, Magnetic shells for gastrointestinal endoscopic capsule as a means to control their motion, Biomed. Pharmacother. 60 (2006) 370-374.

[8] M.Schurr, Facts file on medical and technological background and requirements definition, VECTOR project deliverable D1.1/D1.2

[9] R. Carta, B. Lenaerts, J. Thone, G. Tortora, P. Valdastri, A Menciassi, R. Puers, P. Dario, Wireless power supply as enabling technology towards active locomotion in capsular endoscopy, in: EUROSENSORS XXII Book of abstracts, 2008.

[10] M.E. Rentschler, J. Dumpert, S.R. Platt, S.I. Ahmed, S.M. Farritor, D. Oleynikov, Mobile in vivo camera robots provide sole visual feedback for abdominal exploration and cholecystectomy, Surg. Endosc. 20 (1) (2006) 135-138.

[11] K.J. Lim, J.S. Lee, S.H. Park, S.H. Kang, H.H. Kim, Fabrication and characteristics of impact type ultrasonic motor, J. Eur. Ceram. Soc. 27 (2007) 4159-4162.

[12] J.-D. Hsu, C.-L. Tsai, Y.-Y. Tzou, Design and implementation of a voice-coil motor servo control ic for auto-focus mobile camera applications, in: Power Electronics Specialists Conference, 2007. PESC 2007 IEEE, 2007, pp. 1357-1362.

[13] B. Berge, J. Peseux, Variable focal lens controlled by an external voltage: an application of electrowetting, Eur. Phys. J. E - Soft Matter 3 (2000) 159-163.

[14] L. Saurei, J. Peseux, F. Laune, B. Berge, Tunable liquid lens based on electrowetting technology: principle, properties and applications, in: 10th Annual Micro-optics Conference, 2004.

[15] J. Chen, W. Wang, J. Fang, K. Varahramyan, Variable-focusing microlens with microfluidic chip, J. Micromech. Microeng. 14 (2004) 675-680.

[16] H. Oku, X. Ishikawa, A variable-focus lens with lkhz bandwidth applied to axialscan of a confocal scanning microscope, IEEE (2003) 309-310.

[17] W. Wang, J. Fang, Variable focusing microlens chip for potential sensing applications, Sensors Journal, IEEE 7(1) (2007) 11-17.

[18] S. Sato, Applications of liquid crystals to variable-focusing lenses, Opt. Rev. 6 (1999) 471-485

[19] B. Berge, Liquid lens technology: principle of electrowetting based lenses and applications to imaging, in: Proc. 18th IEEE International Conference on Micro Electro Mechanical Systems MEMS, 2005, pp. 227-230.

[20] R. Shaik, L. Lasinger, F. Krogmann, W. Monch, H. Zappe, Fabrication and characterization of a repositionable liquid micro lens system, in: Proc. IEEE/LEOS International Conference on Optical MEMS and Nanophotonics, 2007, pp. 139-140.

[21] Quilliet, Berge, Investigation of effective interface potentials by electrowetting, Europhys. Lett. 60 (2002) 99-105.

[22] J. Thone, D. Turgis, R. Carta, R. Puers, Design of an inductively powered 2 mbits fsk trasmitter for wireless capsule endoscopy, in: Proceeding of XXII EUROSENSORS, Dresden, Germany, 7-10 September 2008.

[23] P. Valdastri, A. Menciassi, P. Dario, Trasmission power requirements for novel zigbee implants in the gastrointestinal tract, IEEE Trans. Biomed. Eng. 55 (2008) 1705-1710.

[24] E. Susilo, P. Valdastri, A. Menciassi, P. Dario, A miniaturized wireless control platform for robotic capsular endoscopy using pseudokernel approach, in: Proceeding of XXII EUROSENSORS, Dresden, Germany, 7-10 September 2008.

[25] A. Santos, C. Ortiz, D. Solorzano, J. Vaquero, J.M. Pena, N. Malpica, F. Pozo, Evaluation of autofocus functions in molecular cytogenetic analisys, J. Microsc. 188 (1997) 264-272.

[26] L. Firestone, K. Cook, K. Culp, N. Talsania, K. Preston, Comparison of autofocus methods for automated microscopy, Cytometry 12 (3) (1991) 195-206.

[27] J. Brenner, B. Dew, J. Horton, An automated microscope for cytologic research, J. Histochem. Cytochem. 24 (1976) 24-100.

[28] D. Vollath, The influence of the scene parameters and of noise on the behaviour of automatic focusing algorithm, J. Microsc. 151 (1988) 133-146.

[29] N. Kehtarnavaza, H.-J. Ohb, Development and real-time implementation of a rule-based auto-focus algorithm, Real-Time Imaging 9 (2003) 197-203.

[30] L. Saurei, G. Mathieu, B. Berge, Design of an autofocus lens for vga $1 / 4 \mathrm{ccd}$ and cmos sensors, in: SPIE, 2003.

\section{Biographies}

Carmela Cavallotti received her Laurea Degree in biomedical engineering (with Honors) from the Campus Bio-Medico University in Rome in December 2007. Currently she works at the CRIM Lab of the Scuola Superiore Sant'Anna in Pisa.

Marco Piccigallo was born in 1978 in Pisa, Italy. He received the M.Sc. degree in mechanical engineering from University of Pisa, Italy, in 2003 and the Ph.D. in biorobotics at IMT Institute for Advanced Studies Lucca, Italy, in 2008. He joined the CRIM Lab of Scuola Superiore Sant'Anna, Pisa, in 2004. His main research interests are in the field of biomedical robotics and computer-assisted surgery. 
Ekawahyu Susilo received his Bachelor Degree in electrical engineering from Universitas Surabaya, Indonesia in July 2000. Six months later he joined Universitas Surabaya and become lecturer since then. During 4 years time, he had been Head of Automation Laboratory in the same university and also Head of Microcontroller Division in School of Technology (SofT) in Surabaya, Indonesia. In May 2005 he started Ph.D. Study in Scuola Superiore Sant'Anna, Pisa, Italy, in the field of Bioengineering. Now he is in the final year of the Ph.D. Study. His major is in real time embedded system, embedded system design and robotic applications. He has received many awards in scientific writings and robotic contests.

Pietro Valdastri received his Laurea Degree in electronic engineering (with Honors) from the University of Pisa in February 2002. In the same year he joined the CRIM Lab from the University of Pisa in February 2002. In the same year he joined the CRIM Lab
of the Scuola Superiore Sant'Anna in Pisa as Ph.D. student. In 2006, he obtained his Ph.D. in bioengineering from Scuola Superiore Sant'Anna by discussing a thesis titled "Multi-Axial Force Sensing in Minimally Invasive Robotic Surgery". He is now assistant professor at CRIM Lab, with main research interests in the field of implantable robotic systems and active capsular endoscopy. He is working on several European projects for the development of minimally invasive and wireless biomedical devices.

Arianna Menciassi received her Laurea Degree in physics (with Honours) from the University of Pisa in 1995. In the same year, she joined the CRIM Lab of the Scuola Superiore Sant'Anna in Pisa as a Ph.D student in bioengineering with a research program on the micromanipulation of mechanical and biological micro-objects. In 1999, she received her Ph.D. degree by discussing a thesis titled "Microfabricated Grippers for Micromanipulation of Biological and Mechanical Objects". Currently she is a professor of biomedical robotics at the Scuola Superiore Sant'Anna, Pisa. Her main research interests are in the fields of biomedical micro- and nano-robotics, microfabrication technologies, micromechatronics and microsystem technologies. She is working on several European projects and international projects for the development of micro- and nano-robotic systems for medical applications.

Paolo Dario received his Laurea Degree in mechanical engineering from the University of Pisa in 1977. Currently, he is a professor of biomedical robotics at the Scuola Superiore Sant'Anna, Pisa. He also established and teaches the course on mechatronics at the School of Engineering, University of Pisa. He has been a visiting professor at the Ecole Polytechnique Federale de Lausanne (EPFL), Lausanne, Switzerland, and at Waseda University, Tokyo, Japan. He is the director of the CRIM Lab of Scuola Superiore Sant'Anna, where he supervises a team of about 70 researchers and Ph.D. students. His main research interests are in the fields of medical robotics, mechatronics and microengineering, and specifically in sensors and actuators for the above applications. He is the coordinator of many national and European projects, the editor of two books on the subject of robotics and the author of more than 200 journal papers. He is a member of the Board of the International Foundation of Robotics Research. He is an associate editor of the IEEE Transactions on Robotics and Automation, a member of the Steering Committee of the Journal of Microelectromechanical Systems and a guest editor of the Special Issue on Medical Robotics of the IEEE Transactions on Robotics and Automation. He serves as president of the IEEE Robotics and Automation Society and as the co-chairman of the Technical Committee on Medical Robotics of the same society. 